\title{
A SEPARABLE SPACE WITH NO REMOTE POINTS
}

\begin{abstract}
ALAN DOW
ABstract. In the model obtained by adding $\omega_{2}$ side-by-side Sacks reals to a model of $\mathrm{CH}$, there is a separable nonpseudocompact space with no remote points. To prove this it is also shown that in this model the countable box product of Cantor sets contains a subspace of size $\omega_{2}$ such that every uncountable subset has density $\omega_{1}$. Furthermore assuming the existence of a measurable cardinal $\kappa$ with $2^{\kappa}=\kappa^{+}$, a space $X$ is produced with no isolated points but with remote points in $v X-X$. It is also shown that a pseudocompact space does not have remote points.
\end{abstract}

\section{INTRODUCTION}

All spaces are completely regular Hausdorff. For a space $X, \beta X$ is the Stone-Čech compactification of $X$ and $v X \subset \beta X$ is the Hewitt-Nachbin realcompactification. A point $p \in \beta X-X$ is a remote point of $X$ if $p$ is not in the closure of any nowhere dense subset of $X$. A space $X$ is pseudocompact if each locally finite family of open sets is finite (equivalently: if $v X=\beta X$ or if each real-valued continuous function is bounded).

In this article we answer questions of van Douwen's about remote points which are in a sense at opposite ends of the spectrum. The first is whether or not each separable nonpseudocompact space has remote points. In 1960, Fine and Gillman introduced remote points and proved, assuming $\mathrm{CH}$, that each separable nonpseudocompact space has remote points. Van Douwen (and independently Chae and Smith) proved that $\mathrm{CH}$ was not needed if one assumed, in addition, that the space had a countable $\pi$-base (e.g. if it is metrizable). It is asked in [vDvM] if all nonpseudocompact separable spaces have remote points. Various partial results have been obtained; i.e. Martin's Axiom (MA) can replace $\mathrm{CH}$, countable $\pi$-weight can be weakened to $\pi$-weight at most $\omega_{1}$ and in each of these cases separable could be replaced by ccc (no uncountable pairwise disjoint collections of open sets) plus weight at most c. Also any product (nonpseudocompact) of metric spaces has remote points [DP]. It has also been shown that if $\mathrm{CH}$ is assumed, then separable can be weakened to the space

Received by the editors March 21, 1987 and, in revised form, November 6, 1987.

1980 Mathematics Subject Classification (1985 Revision). Primary 54D35, 54D40, 54D60, 54A35; Secondary 03E35, 03E55.

Key words and phrases. Remote points, measurable cardinals, side-by-side Sacks forcing.

Supported by the NSERC of Canada. 
having only c real-valued continuous functions [KvMM]. Examples of nonpseudocompact spaces without remote points were produced [vDvM, KvMM and D3] but these were all far from being separable. In $\S 5$, we show that it is consistent (with ZFC) to have a separable nonpseudocompact space with no remote points.

In each of the above results we are assuming that the space in question is nonpseudocompact. In 1979, Terada showed (with an unnecessary assumption about measurable cardinals) that a pseudocompact space does not have remote points. In fact Terada showed that if the cellularity of $X$ is less than the first measurable cardinal then no point of $v X-X$ is a remote point of $X$ (this was improved by Blair to include those $X$ whose closed screenable subspaces had size less than the first measurable cardinal). The question of the existence of remote points is really only interesting for those $X$ with no isolated points since every point of $\beta D-D$ is remote for discrete $D$ (see 6.5 below). In [vD2], van Douwen asks if there is a space with no isolated points having a remote point in $v X-X$. We show that this statement is equiconsistent with the existence of a measurable cardinal. We also substantially improve Terada's and Blair's results by finding a much wider class of spaces which do not have remote points in $v X-X$.

\section{WHY STUDY REMOTE POINTS?}

The study of remote points began, most probably, simply as an effort to better understand $\beta X$. The property of a remote point at first encounter is a bit mystifying (at least to the author) in that you have a point in the closure of a set and yet its neighborhood trace never really settles down on any "topologically small" set of points. It was then noticed that a remote point can also be thought of as a point in $E(\beta X)$ (see [W5]) and this led to their usage in the study of coabsolutes [G1,2,3, PoW and W2,3,4]. It was van Douwen who first discovered their role in the study of the nonhomogeneity of $\beta X$ and $\beta X-X$ and van Mill applied the techniques of their construction to constructing other points in $\beta X$ [vD1,2, vM1,2, VWa]. Frolik had proven that $\beta X$ (for nonpseudocompact $X$ ) is never homogeneous but his proof does not give any insight as to why, in that it does not produce topologically interesting points. Remote points are such points, as are the better known $P$-points [R] and weak $P$-points [K2]. In [vM2], van Mill, relying on earlier work in [vM1, vD2, K2, Be, DvM and D1], proved, assuming MA, that every nonpseudocompact space $X$ has a point in $\beta X$ which is not the limit of any countable nowhere dense subset of $\beta X$ (call such a point of weak remote point). MA was actually only required for the "small" spaces (ccc plus weight at most c), in particular the separable spaces. The example we produce is a separable space with no remote points but we do not know if it has weak remote points (see 2.4).

Besides having applications in topology, the study of remote points has been both a consumer and producer of combinatorial set theory and consistency results. This is not so surprising given that the translation of the notion of 
a remote point to that of an ultrafilter on a Boolean algebra (2.1) looks very much like the definition of a $P$-point ultrafilter on $\mathscr{P}(\omega) /$ fin. As van Mill observes in [vM2], a technique developed for constructing remote points and weak $P$-points is to construct, say, $n$-linked remote collections (e.g. see 2.2 below), rather than a filter, and then piece these collections together to build a filter (2.3). However it is precisely these $n$-linked remote collections which have found an application in forcing (see [DTW, F1, 2, T] or 2.7). As an example of it being a producer of consistency results see $\S 4$ where we prove a result about the box product of Cantor sets.

So what is left to do? It will undoubledly turn out to be a very interesting solution if one ever determines whether or not it is consistent (with or without large cardinals) that every nonpseudocompact ccc space (or even countable space) has remote points. It is also still open whether or not every nonpseudocompact space has a weak remote point or an $\omega$-far point [vD2]. Furthermore the applications to forcing should be explored where remote collection is generalized to that of a lynx or endowment (see [DTW, F1, 2, T] or 2.2 below), including finding posets possessing various lynxes [D5].

\section{Preliminaries}

Our reference for facts about $\beta X$ and $v X$ is [GJ]. Recall that $C(X)\left(C^{*}(X)\right)$ is the ring of all real-valued continuous (bounded) functions on $X$. A subset $Z$ of $X$ is a zero set if $Z=Z(f)=f^{-1}(0)$ for some $f \in C(X)$; a cozero set is the complement of a zero set. Let $\mathscr{Z}(X)$ be the lattice of all zero sets of $X$. The topological space $\beta X$ is the space of $\mathscr{Z}$-ultrafilters on $X$ and we identify $X$ with the set of fixed ultrafilters. The topology is given by $\operatorname{cl}_{\beta X} Z=Z \cup\{p \in$ $\beta X-X: Z \in p\}$ for each $Z \in \mathscr{Z}(X)$; in particular disjoint zero sets have disjoint closures. The space $v X$ is $\{p \in \beta X: p$ is countably complete $\}$. For any $f \in C^{*}(X)(C(X))$ there is a unique function $\beta f \in C^{*}(\beta X)(v f \in C(v X))$ such that $f \subset v f \subset \beta f$. A pair of subsets of $X$ is said to be completely separated if there is a function in $C(X)$ sending all members of one set to 0 and the other set to 1 (equivalently if they are contained in disjoint zero sets or if they have disjoint closures in $\beta X$ ).

A collection $\mathscr{F} \subset \mathscr{Z}(X)$ is called remote if for each nowhere dense set $D \subset X$ there is a $Z \in \mathscr{F}$ such that $Z$ and $D$ are completely separated. Hence a remote point in $\beta X-X$ is a remote free ultrafilter on $\mathscr{Z}(X)$. The Souslin number of $X, \hat{c}(X)$, is the least cardinal such that all families of pairwise disjoint open sets have smaller cardinality. If $\hat{c}(X) \leq \omega_{1}$ then $X$ is ccc. For a family $\left\{X_{\alpha}: \alpha \in I\right\}$ of spaces, let $\sum_{\alpha \in I} X_{\alpha}$ denote the space $\bigcup\left\{\{\alpha\} \times X_{\alpha}: \alpha \in I\right\}$ where $\{\alpha\} \times X_{\alpha}$ is open and homeomorphic to $X_{\alpha}$ for each $\alpha \in I$. A space $X$ is 0-dimensional if $C O(X)$, the collection of clopen sets, forms a basis for the topology. If $B$ is any Boolean algebra then the Stone space of $B$, or the space of ultrafilters, $S(B)$, is a compact 0-dimensional space and $C O(S(B)) \cong B$ (where $b^{*}=\{\mathscr{U} \in S(B): b \in \mathscr{U}\} \in C O(S(B))$ for $b \in B$ ). If $\left\{B_{\alpha}: \alpha \in I\right\}$ is 
a collection of Boolean algebras then let $\sum_{\alpha \in I} B_{\alpha}$ denote the Boolean algebra $C O\left(\sum_{\alpha \in I} S\left(B_{\alpha}\right)\right)$ or the corresponding algebra generated by $\bigcup\left\{\{\alpha\} \times B_{\alpha}: \alpha \in I\right\}$, and if $P$ is a separative poset let $\sum_{I} P$ denote $\sum_{\alpha \in I} B_{\alpha}$ where each $B_{\alpha}$ is the unique complete Boolean algebra containing $P$ densely. A set $A \subset P, P$ a poset, is said to be predense if for each $p \in P$ there is an $a \in A$ compatible with $p$ (equivalently the set $\{p \in P: \exists a \in A p \leq a\}$ is dense in $P$ ).

Definition 2.1. For any collection $\left\{B_{\alpha}: \alpha \in I\right\}$ of Boolean algebras, we call $\mathscr{F} \subset \sum_{\alpha \in I} B_{\alpha}$ remote if for each sequence $\left\{A_{\alpha}: \alpha \in I\right\}$ with each $A_{\alpha}$ a predense subset of $B_{\alpha}$, there are $A_{\alpha}^{\prime} \in\left[A_{\alpha}\right]^{<\omega}$ such that $\bigvee_{\alpha \in I} \bigvee A_{\alpha}^{\prime} \in \mathscr{F}$.

Lemma 2.2. For any collection $\left\{B_{\alpha}: \alpha \in I\right\}$ of Boolean algebras, $X=\sum_{\alpha \in I} S\left(B_{\alpha}\right)$ has a remote point iff $\sum_{\alpha \in I} B_{\alpha}$ has a remote filter.

Proof. If $\mathscr{F} \subset \sum_{\alpha \in I} B_{\alpha}$ is remote as in 2.1, then the collection $\mathscr{F}^{*}=\left\{b^{*}: b \in\right.$ $\mathscr{F}\} \subset C O(X) \subset \mathscr{Z}(X)$ is remote. Hence if $\mathscr{F}$ is a filter then $\mathscr{F}^{*}$ is a filter base on $\mathscr{Z}(X)$. Now suppose $p \in \beta X-X$ is a remote filter. Let $\mathscr{F}^{*}=p \cap C O(X)$ and define $\mathscr{F}=\left\{b \in \sum_{\alpha \in I} B_{\alpha}: b^{*} \in \mathscr{F}^{*}\right\}$. Clearly $\mathscr{F}$ is a filter so let us check that it is remote. Let $\left\{A_{\alpha}: \alpha \in I\right\}$ be a sequence of predense sets as in 2.1. Let $D \subset X$ be defined as $\bigcup_{\alpha \in I}\left[S\left(B_{\alpha}\right)-\bigcup\left\{a^{*}: a \in A_{\alpha}\right\}\right]$. Since each $A_{\alpha}$ is predense in $B_{\alpha}, D$ is a nowhere dense subset of $X$. Choose $Z \in p$ with $Z \cap D=0$ and note that for each $\alpha \in I, Z \cap S\left(B_{\alpha}\right)$ is a compact subset of $\bigcup\left\{a^{*}: a \in A_{\alpha}\right\}$. Therefore we may choose $A_{\alpha}^{\prime} \in\left[A_{\alpha}\right]^{<\omega}$ for $\alpha \in I$ so that $Z \subset \bigcup_{\alpha \in I} \bigcup\left\{a^{*}: a \in A_{\alpha}^{\prime}\right\} \in \mathscr{F}^{*}$.

There are interesting generalizations of the notion of remote filter. Indeed let us say that $\mathscr{F} \subset \mathscr{Z}(X)$ is a weak remote collection if it satisfies the definition of remote after "nowhere dense" is replaced by "separable nowhere dense". A collection $\mathscr{F} \subset \mathscr{P}(X)$ is n-linked for $n \in \omega$ if $\cap \mathscr{F}^{\prime} \neq 0$ for any $\mathscr{F}^{\prime} \in$ $[\mathscr{F}]^{\leq n}$. Now we have the notions (weak) remote $n$-linked collection on $X$ and weak remote filter on $X$. Similarly for a Boolean algebra $B$ we define $\mathscr{F} \subset B$ as a (weak) remote $n$-linked collection if $\mathscr{F}^{*}=\left\{b^{*}: b \in \mathscr{F}\right\}$ is the corresponding thing on $S(B)$. A collection $\mathscr{F}$ of subsets of $\sum_{n \in \omega} X_{n}$ is called nice $[\mathrm{vM}]$ if $\left\{n \in \omega: F \cap X_{n}=0\right\}$ is finite for each $F \in \mathscr{F}$. A generalization of remote that has its origins in forcing is that of a lynx [F2]. For a complete Boolean algebra $B, \mathscr{L} \subset B$ is a $(\kappa, \lambda)-n$-lynx if for each predense $A \subset B$ with $|A|<\kappa$ there is an $A^{\prime} \in[A]^{<\lambda}$ with $\bigvee A^{\prime} \in \mathscr{L}$ and $\mathscr{L}$ is $n$-linked. The notion of $n$-linked arose in the context of remote points (e.g. [vD2 and CS]) because as van Mill [vM2] points out it is easier to construct $n$-linked collections than filters and the following holds.

Theorem 2.3 [vM2]. If, for $n \in \omega, X_{n}$ has an $n$-linked (weak) remote collection, then $\sum_{n \in \omega} X_{n}$ has a nice (weak) remote filter.

Remark 2.4. Van Mill asks if $X=\sum_{n \in \omega} X_{n}$ always has a nice weak remote filter (since if it does it then follows from [vM2] that $\beta X$ has a weak remote point). It appears that the space in $[D 2,4]$ can be modified slightly to prove that 
the answer is consistently no. However the really interesting question in this case is whether $\beta X$ has a weak remote point; i.e. one would like to produce an example with no weak remote filter.

Let us now record some known results about remote collections.

Proposition 2.5. (a) Each metric space has remote $n$-linked collections for any $n \in \omega$ [vD2, CS], but there is a separable space with no remote 2-linked collection [D2].

(b) $M A$ implies $B=\sum_{n \in \omega} B_{n}$ has a nice remote filter providing $B$ is ccc and $|B|=\mathbf{c}[\mathrm{D} 1]$.

(c) The space $\omega \times U\left(\omega_{2}\right)(U(\kappa)$ is the subspace of $\beta \kappa$ consisting of all uniform ultrafiters) has no remote points and there is a space of weight $\omega_{2}$ with no remote points [vDvM, KvMM].

(d) Any nonpseudocompact ccc space with $\pi$-weight less than $\kappa_{0}$ (defined below) has remote points (providing the covering lemma holds) [D4].

For any $p \in \omega^{*},\left({ }^{\omega} \omega,<_{p}\right)$ is a linear order (modulo equivalence classes) where for $f, g \in{ }^{\omega} \omega, f<{ }_{p} g$ iff $\{n \in \omega: f(n)<g(n)\} \in p$. Let $\kappa_{0}$ be the least cardinal such that $\left({ }^{\omega} \omega,<_{p}\right)$ has a cofinal sequence of cardinality less than $\kappa_{0}$ for all $p \in \omega^{*}$. The covering lemma is not needed in (d) for $\pi$-weight $\omega_{1}$ (and $\kappa_{0}$ is always greater than $\omega_{1}$ ). We include (d) because it seems to indicate that in order to produce a model by forcing in which there is a separable space with no remote points we must use an ${ }^{\omega} \omega$-bounding poset (see 3.2 below).

We end this section by proving a result (2.7) which show how lynxes can be used in forcing arguments which has as a consequence that certain spaces have no remote points (in fact $\omega \times U\left(\omega_{1}\right), \omega \times U\left(\omega_{2}\right)$ and even a space with a dense metrizable subspace). We begin with a trivial observation.

Lemma 2.6. If $B$ is a Boolean algebra such that $\sum_{\omega} B$ has remote points then $B$ has an $\left(\infty, \omega_{1}\right)$-2-lynx (where $\infty$ can be replaced by $\left.|B|^{+} \cdot \omega\right)$.

We say that a poset $P$ has a $(\kappa, \lambda)$-2-lynx below $p \in P$ if the Boolean algebra generated by $\{q \in P: q \leq p\}$ has a $(\kappa, \lambda)$-2-lynx.

Proposition 2.7. Let $P$ be a poset which has an $\left(\infty, \omega_{1}\right)$-2-lynx below $p$ for each $p \in P$ and let $X$ be a space with a subset $D$ consisting of $P$-points in $X$. If, after forcing with $P$, the points of $D$ can be surrounded by disjoint neighborhoods (i.e. separated) then $D$ is separated in the ground model. ( $A$ point is a P-point if the intersection of countably many neighborhoods is still a neighborhood.)

Proof. For $d \in D$, let $\{B(d, \alpha): \alpha<\lambda\}$ be a neighborhood base for $d$. Let $\dot{g}$ be a forcing term and let $p \in P$ be chosen so that $p \Vdash$ "for each $d \in \check{D}$, $\dot{g}(d) \in \grave{\lambda}$ and $\check{B}(d, \dot{g}(d)) \cap \check{B}(e, \dot{g}(e))=0$ for $d \neq e \in \check{D}$ ". Fix $\mathscr{L} \subset$ $[\{q \in P: q<p\}]^{\leq \omega}$ such that $\{\bigvee A: A \in \mathscr{L}\}$ generates an $\left(\infty, \omega_{1}\right)$-2-lynx below $p$ (i.e. for each $A$ predense below $p$ there is an $A^{\prime} \in[A]^{\leq \omega}$ such that $A^{\prime} \in \mathscr{L}$ and for $A_{0}, A_{1} \in \mathscr{L}$ there are $a_{0} \in A_{0}$ and $a_{1} \in A_{1}$ such that $a_{0}$ and 
$a_{1}$ are compatible). Now, for each $d \in D$, the set $A_{d}=\{q<p: \exists \alpha<\lambda q \Vdash$ " $\dot{g}(d)=\alpha$ " $\}$ is predense below $q$, hence there is a countable $A_{d}^{\prime} \in \mathscr{L} \cap\left[A_{d}\right]^{\leq \omega}$. For $d \in D$, let $f(d) \in \lambda$ be chosen so that $q \Vdash$ " $\breve{B}(d, f(d)) \subset \check{B}(d, \dot{g}(d))$ " for all $q \in A_{d}^{\prime}$ (since $d$ is a $P$-point). Now $B(d, f(d)) \cap B(e, f(e))=0$ for all $d \neq e \in D$ since we may choose $q \in A_{d}^{\prime}, q^{\prime} \in A_{e}^{\prime}$ and an $r<q, q^{\prime}$. Hence $r \Vdash$ “ $\breve{B}(d, f(d)) \cap \check{B}(e, f(e)) \subset \check{B}(d, \dot{g}(d)) \cap \check{B}(e, \dot{g}(e)) \neq 0$ ”.

Corollary 2.8. Let $X$ be any one of the spaces $U\left(\omega_{1}\right), U\left(\omega_{2}\right)$ or $(D(\kappa)+1)^{\omega}$ (where $D(\kappa)+1$ is the one-point compactification of the discrete space $\left.\kappa \geq \omega_{2}\right)$. Then $\omega \times X$ has no remote points.

Proof. Clearly $\omega \times X$ has remote points iff $\sum_{n \in \omega} C O(X)$ has a remote filter. Hence to prove the result, it suffices by 2.6 and 2.7 to prove that $P=$ $C O(X)-\{0\}$ does not satisfy the conclusion of 2.7. Now, it is known that forcing with $P$ introduces a function from $\omega$ onto $\omega_{2}$. Therefore, by some simple topology it suffices to find a $P$-space with a closed discrete set of size $\omega_{2}$ which cannot be separated. Finally $\omega_{1} \cup \mathscr{A}$ is such a space where $\mathscr{A}$ is a maximal family of uncountable subsets of $\omega_{1}$ with pairwise countable intersection and a neighborhood base for $A \in \mathscr{A}$ is $\left\{\{A\} \cup A-\alpha: \alpha<\omega_{1}\right\}$ and $\omega_{1}$ is open and discrete.

\section{THE MODEL}

In this section we introduce the forcing and the model that we will use in $\S 5$ to produce our space. The forcing is side-by-side Sacks forcing over a model of $\mathrm{CH}$, which was introduced by Baumgartner [B1,2]. For the basics of forcing see [K1 or B3] and [ChKe] for model theory. We shall adopt the notation in [B1,2] and in this section list the basic results about the forcing found in [B2] or 6.4 of [B1]. Although [B2] has not yet been published the techniques involved are very similar to those found in [BL or S] concerning $\omega$-proper, ${ }^{\omega} \omega$-bounding iterations, hence we do not see any need to include the proofs of 3.1 and 3.2.

Let $S q=\left\{{ }^{n} 2: n<\omega\right\}$. A nonempty $p \subset S q$ is called a perfect tree iff

(1) $\forall s \in p \forall n<\omega s \mid n \in p$ and

(2) $\forall s \in p \exists t, u \in p s \subset t, s \subset u$ and $t, u$ are incomparable.

PS is the poset of all perfect trees ordered by $p \leq q$ iff $p \subset q$. If $G$ is $P S$ generic over $V$ (the ground model) then $f_{G}=\bigcup\{s \in S q: \forall p \in G s \subset p\} \in{ }^{\omega} 2$ and $f_{G} \notin V$. We call $f_{G}$ the Sacks real added by $P S$. Let $P S\left(\omega_{2}\right)$ be the set of functions $p$ with domain a countable subset of $\omega_{2}$ and range $P S$. Define $p \leq q$ for $p, q \in P S\left(\omega_{2}\right)$ if domain $(q) \subset$ domain $(p)$ and, for all $\alpha \in$ domain $(q), p(\alpha) \leq q(\alpha)$. We shall let $x_{\alpha}$ be the Sacks real added (in the above sense) by the $\alpha$ th copy of $P S$; i.e. $x_{\alpha}=\bigcup\{s \in S q: \forall p \in G \alpha \in \operatorname{domain}(p)$ implies $s \subset p(\alpha)\}$.

Theorem 3.1 $(\mathrm{CH}) . \quad P S\left(\omega_{2}\right)$ has the $\omega_{2}$-chain condition.

Theorem 3.2. If $p \in P S\left(\omega_{2}\right)$ and $p \Vdash$ " $\dot{f}: \omega \rightarrow V$ ", then there are $q \leq p$ and $a$ sequence of finite sets $\left\{F_{n}: n<\omega\right\}$ such that $q \Vdash$ " $\forall n \dot{f}(n) \in \breve{F}_{n}$ ". 
Both 3.1 and 3.2 are proven in [B1,2] and the proof of 3.2 is quite lengthy. A forcing poset $P$ is said to be ${ }^{\omega} \omega$-bounding if for each $f \in{ }^{\omega} \omega \cap V[G]$ there is a $g \in{ }^{\omega} \omega \cap V$ such that $f(n)<g(n)$ for all $n<\omega$ (where $G$ is any $P$-generic filter over $V$ ).

Corollary $3.3(\mathrm{CH})$. PS $\left(\omega_{2}\right)$ preserves all cardinalities, cofinalities and is ${ }^{\omega} \omega$ bounding. Furthermore, if $G$ is $P S\left(\omega_{2}\right)$-generic over $V$, then $V[G]$ satisfies $\mathbf{c}=\omega_{2}^{V}$.

\section{A RESULT ON BOX PRODUCTS}

We prove that in the model from $\S 3$ the countable box product of Cantor sets has a property we shall need for the example produced in $\S 5$. Let us let $\square C$ denote the box topology on ${ }^{\omega} C$ (see [vD3, Ru or Wi]). For any $s \in S q$, let $[s]=\left\{x \in C={ }^{\omega} 2: s \subset x\right\}$. Hence for any $f \in{ }^{\omega} S q,[f]=\prod_{n \in \omega}[f(n)]$ is a clopen subset of $\square C$. For any $y \in{ }^{\omega} C$ and $f \in{ }^{\omega} \omega$, let $\left[y^{f}\right]=\left[f^{\prime}\right]$ where $f^{\prime} \in{ }^{\omega} S q$ is defined by $f^{\prime}(n)=y \mid f(n)$.

Let $V$ be a model satisfying $\mathrm{CH}$ and let $G$ be $P S\left(\omega_{2}\right)$-generic over $V$. We shall prove that the following statement holds in $V[G]$ :

"there is a subspace $Y$ of $\square C$ such that $|Y|=\mathrm{c}$ and for all (*) $\quad Z \subset Y$, the density of $Z$ and the Lindelöf degree of $Z$ are both equal $\min \left\{|Z|, \omega_{1}\right\} "$.

Recall that the density of a space is the minimum cardinality of a dense subset and the Lindelöf degree is the minimum cardinal $\kappa$ such that every open cover has a subcover of size at most $\kappa$.

It is trivial that (*) follows from $\mathrm{CH}$ since the subspace of $\square C$ consisting of constant functions is closed and discrete. Furthermore if $(*)$ holds then $d=\omega_{1}$ (recall that $\mathbf{d}$ is the minimum cardinality of a dominating family in ${ }^{\omega} \omega$ ). To see this note first of all that if $Y$ exhibits that $(*)$ holds and $y \in Y$ then $\{x \in Y:\{n: x(n) \neq y(n)\}$ is finite $\}$ is a separable hence countable subset of $Y$. Next we observe that if $Y \subset \square C,|Y|<\mathrm{d}$ and $x \in \square C-Y$ is such that for each $y \in Y\{n: y(n) \neq x(n)\}$ is infinite then $x$ is not in the closure of $Y$. Indeed, we may choose $h \in{ }^{\omega} \omega$ so that for each $y \in Y\left\{n:\left.x(n)\right|_{h(n)} \neq\left. y(n)\right|_{h(n)}\right\}$ is infinite and therefore $\left[x^{f}\right]$ is disjoint from $Y$. Now $d=\omega_{1}$ follows from (*) since a dense subset of $Y$ must have cardinality $\mathbf{d}$. However $\mathbf{d}=\omega_{1}$ does not imply (*) (see 4.4). For $\lambda \in \lim \left(\omega_{2}\right)$, define $y_{\lambda} \in \square C$ by $y_{\lambda}(n)=x_{\lambda+n}$ (in $V[G])$ and let $Y=\left\{y_{\lambda}: \lambda \in \lim \left(\omega_{2}\right)\right\}$. For the rest of this section we must work in $V$.

Theorem 4.1. Let $\dot{Z}$ be a PS( $\left.\omega_{2}\right)$-term such that $1 \Vdash$ " $\dot{Z} \subset \dot{Y}=\left\{\dot{y}_{\lambda}: \lambda \in\right.$ $\left.\lim \left(\omega_{2}\right)\right\}$ and $|\dot{Z}|=\omega_{2}$ ". Then there is a $\alpha \in \omega_{2}$ such that, for all $\delta \in \omega_{2}-\alpha$, $1 \Vdash$ " $\dot{Z} \cap\left\{\dot{y}_{\lambda}: \lambda \in \lim (\delta)\right\}$ is dense and not open in $\dot{Z}$ ".

Proof. Let $\alpha \in \omega_{2}$ with $\operatorname{cf}(\alpha)=\omega_{1}$ be such that there is an elementary submodel $M$ of $H\left(\omega_{3}\right)$ (see [K1]) such that $|M|=\omega_{1},\left\{P S\left(\omega_{2}\right), \dot{Z}, C\right\}$ and 
$P S(\omega)$ are all subsets of $M$, and $M \cap \omega_{2}=\alpha$. Let $M$ be such a model, $\alpha=M \cap \omega_{2}$, and note that $P S(\alpha) \subset M$. Suppose $\lambda \in \lim \left(\omega_{2}\right)$ and $p \in P S\left(\omega_{2}\right)$ are such that $p \Vdash$ " $\dot{y}_{\lambda} \in \dot{Z}$ and $\dot{y}_{\lambda}$ is not a limit point of $\dot{Z} \cap\left\{\dot{y}_{\beta}: \beta \in \lim (\alpha)\right\}$ ". Since $P S\left(\omega_{2}\right)$ is ${ }^{\omega} \omega$-bounding, there is an $f \in{ }^{\omega} \omega$ (in $V$ ) and a $q \leq p$ such that $q \Vdash$ " $\left[\dot{y}_{\lambda}^{f}\right] \cap \dot{Z} \cap\left\{\dot{y}_{\beta}: \beta \in \lim (\alpha)\right\}=0$ ". Let $q_{0}=q \cap M$ and note that $q_{0}$ has the property " $\exists q \leq q_{0} \exists \lambda \in \lim \left(\omega_{2}\right) q \Vdash$ " $\dot{y}_{\lambda} \in \dot{Z}$ and $\dot{y}_{\lambda} \in\left[f^{\prime}\right]$ " " where we may assume $f^{\prime} \in{ }^{\omega} S q$ and $q \Vdash{ }^{\prime} "\left[\dot{y}_{\lambda}^{f}\right]=\left[f^{\prime}\right] "$ since this only requires that $q$ "decides" $x_{\lambda+n} \uparrow f(n)$ for $n<\omega$. Therefore, by elementarity and the fact that $\left\{q_{0}, f^{\prime}\right\} \subset M$, there is a $q_{1} \in M$ with $q_{1} \leq q_{0}$ and a $\beta \in M$ with $\beta \in \lim (\alpha)$ such that $q_{1} \Vdash$ “ $\dot{y}_{\beta} \in \dot{Z}$ and $\dot{U}_{\beta} \in\left[f^{\prime}\right]$ ". However, since $\operatorname{PS}\left(\omega_{2}\right)$ is just product forcing, $q_{1}$ is compatible with $q$ and $q_{1} \wedge q \Vdash$ " $\left[\dot{y}_{\lambda}^{f}\right]=\left[f^{\prime}\right]$ and $\left[f^{\prime}\right] \cap \dot{Z} \cap\left\{\dot{y}_{\beta}: \beta \in \lim (\alpha)\right\} \neq 0$ ", which contradicts our assumptions on $p$ and $\lambda$. Therefore $1 \Vdash$ “ $\dot{Z} \cap\left\{\dot{y}_{\beta}: \beta \in \lim (\alpha)\right\}$ is dense in $\dot{Z}$ ”.

Now we show that $1 \Vdash$ " $\dot{Z}-\left\{\dot{y}_{\beta}: \beta \in \lim (\delta)\right\}$ is not closed in $\dot{Z}$ " for any $\delta \in \omega_{2}-\alpha$. Let $J=\left\{\lambda \in \lim \left(\omega_{2}\right)-\delta: \exists q \in P S\left(\omega_{2}\right) q \Vdash\right.$ “ $\left.\dot{y}_{\lambda} \in \dot{Z} "\right\}$ and for each $\lambda \in J$ fix a $q_{\lambda}$ exhibiting that $\lambda \in J$. Since $\mathrm{CH}$ holds in $V$, there are $R \in\left[\omega_{2}\right]^{\omega}, r_{0} \in P S(\omega), r_{1} \in P S(R)$ and $J_{0} \in[J]^{\omega_{2}}$ such that $R$ is the root of the $\Delta$-system $\left\{\operatorname{dom}\left(q_{\lambda}\right): \lambda \in J_{0}\right\}$ and for each $\lambda \in J_{0} q_{\lambda} \cap P S(R)=r_{1}$ and $q_{\lambda} \cap P S([\lambda, \lambda+\omega))$ is isomorphic to $r_{0}$. Let $q_{0}=r_{1} \cap M$. As above, using elementarity, we may choose $q_{1} \in P S\left(\omega_{2}\right) \cap M, q_{1} \leq q_{0}$ and $\beta \in M$ such that $q_{1} \Vdash{ }^{\prime} \dot{y}_{\beta} \in \dot{Z}$ " and $q_{1} \cap P S([\beta, \beta+\omega))$ is isomorphic to $r_{0}$.

Now suppose $f \in{ }^{\omega} \omega \cap V$ and $q_{2} \in P S\left(\omega_{2}\right)$ are such that $q_{2}<q_{1} \wedge r_{1}$ and $q_{2} \Vdash$ " $\left[\dot{y}_{\beta}^{f}\right] \cap \dot{Z}-\left\{\dot{y}_{\gamma}: \gamma \in \lim (\delta)\right\}=0$ ". Without loss of generality we may also assume that there is an $f^{\prime} \in{ }^{\omega} S q \cap V$ such that $q_{2} \Vdash{ }^{\prime}\left[\dot{y}_{\beta}^{f}\right]=\left[f^{\prime}\right]$. Choose $\lambda \in J_{0}$ such that $\operatorname{dom}\left(q_{\lambda}\right) \cap \operatorname{dom}\left(q_{2}\right) \subset R$. Since both $q_{1} \cap P S([\beta, \beta+\omega))$ and $q_{\lambda} \cap P S([\lambda, \lambda+\omega))$ are isomorphic to $r_{0}$ we may choose $q_{3} \leq q_{\lambda} \wedge q_{2}$ so that $q_{3} \Vdash "\left[\dot{y}_{\lambda}^{f}\right]=\left[f^{\prime}\right] "$. In which case $q_{3} \Vdash y_{\lambda} \in$ “[ $\left.\dot{y}_{\beta}^{f}\right] \cap \dot{Z} "$.

Corollary 4.2. In $V[G]$, the space $Y=\left\{y_{\lambda}: \lambda \in \lim \left(\omega_{2}\right)\right\}$ exhibits that (*) holds.

Proof. First observe that countable subsets of $Y$ are closed in $Y$ since we are using the box topology and distinct elements of $Y$ differ on every coordinate. Therefore it suffices to show that $Y$ has hereditary density and Lindelöf degree $\leq \omega_{1}$. Now for density this follows immediately from 4.1. Also if there were a subspace $Z$ with Lindelöf degree $\omega_{2}$, then there would be such a $Z$ which had a cover by open subsets with cardinality $\omega_{1}$. In which case we should have that $Z \cap\left\{y_{\lambda}: \lambda \in \lim (\alpha)\right\}$ is open in $Z$ for each $\alpha \in A^{\prime}$ for some cub $A^{\prime}$. Therefore by 4.1 there is no such $Z$.

Corollary 4.3. Let $g \in{ }^{\omega} \omega \cap V$ be a 1-1 function and redefine $\dot{y}_{\lambda}$ so that $\dot{y}_{\lambda}(n)=\dot{x}_{\lambda+g(n)}$. For any $h \in{ }^{\omega} S q \cap V 1 \Vdash " \dot{Y} \cap[h]$ has cardinality $\omega_{2}$ and for any $Z \subseteq \dot{Y}$ with $|Z|=\omega_{2}, \bar{Z} \cap\left\{\dot{y}_{\lambda}: \lambda \in \lim \left(\omega_{1}\right)\right\} \neq 0$ ”. 
Proof. The redefinition of $\dot{Y}$ makes no difference in the proof of 4.1 since $g$ is in $V$. The proof that $1 \Vdash "|\dot{Y} \cap[h]|=\omega_{2}$ " for $h \in{ }^{\omega} S q \cap V$ is trivial. For the final fact we observe that the role of $M$ (hence $\alpha$ ) besides being uncountable was to find a $\beta$ so that $q_{1} \Vdash$ " $\dot{y}_{\beta} \in \dot{Z}$ ". However in this case with $\left\{q_{\lambda}: \lambda \in J_{0}\right\}$, $R, r_{0}$ and $r_{1}$ as in 4.1 (with the new $\dot{Y}$ ) we may choose any $\beta \in \lim \left(\omega_{1}\right)$ so that $[\beta, \beta+\omega) \cap R=0$ and find $q_{1}<r_{1}$ with $q_{1} \cap P S([\beta, \beta+\omega))$ isomorphic to $r_{0}$. Then proceed as in 4.1 .

Remark 4.4. It can be shown that $(*)$ does not hold if one starts with a model of $\mathrm{CH}$ and adds random reals or uses any proper iteration of the usual kind of reals (e.g. random, Cohen, Sacks, Laver, Mathias, etc.) (see [S]). Let us sketch a proof for the case when Sacks reals are iterated. Assume that $Y$ exhibits that (*) holds and let $D$ be a size $\omega_{1}$ dense subspace of $Y$. In each of the above forcings we may assume that $D$ is in the ground model, $V$. For each $y \in Y$ the set of members of $Y$ which equal $d$ on all but finitely many coordinates is separable hence countable. Therefore for any inner model of $\mathrm{CH}$ the set of members of $Y$ which agree with some member of this model on all but finitely many coordinates has size at most $\omega_{1}$. Furthermore, since $\mathbf{d}=\omega_{1}$, we can assume that the members of ${ }^{\omega} \omega$ in the ground model are dominating. Let $y \in Y$ be chosen so that $y$ disagrees with any member of the ground model on infinitely many coordinates. Let $\alpha$ be the minimum ordinal such that there is a $P_{\alpha}$-name for $y$ and we may assume that we are working in a model after adding some more reals to $V$. Let $M$ be a countable elementary submodel with $y$ etc. in $M$ and let $f \in{ }^{\omega} \omega \cap V$ dominate ${ }^{\omega} \omega \cap M$. Recursively build a tree of conditions $\left\{p_{s}: s \in{ }^{\omega} 2\right\} \subset P_{\alpha} \cap M$ so that $p_{s}$ decides $\left.y(|s|)\right|_{f(n)}$ and, if possible, $p_{s \sim 0}$ and $p_{s>1}$ decide differently. Also if $p_{s}$ can be chosen so that for some $k$ and any $n>\left.|s| y(n)\right|_{f(n)}$ can be decided without strengthening $\left.p_{s}\right|_{p_{\beta_{k}}}$ then choose it so for the next possible $k$ (where $\left\{\beta_{n}: n \in \omega\right\}$ is cofinal in $\alpha$ ). Now if for some $p_{s}$ there is no $k$ as above then by genericity of $\left.p_{s}\right|_{p_{\beta_{k}}}\left[y^{f}\right]$ will not be in $V$. Furthermore in case such a $k$ can always be found then for each $h \in{ }^{\omega} 2$ the meet of $\left\{p_{s}: s \subset h\right\}$ will be a condition. In this case we can assume for some $h$ the corresponding condition will also force that $\left[y^{f}\right]$ is not in $V$ (i.e. we are working in a model in which there are no perfect sets consisting entirely of reals from $V$ ). Therefore in either case $\left[y^{f}\right]$ is a neighborhood of $y$ which misses $D$.

\section{THE SPACE}

In this section we shall show that in the model produced in $\S 3$ there is a separable nonpseudocompact space with no remote points. In fact we shall produce a poset $P$ which is $\sigma$-centered and such that $\sum_{\omega} P$ does not have a remote filter as in 2.1 . 
Definition 5.1. For any set $X \subset C={ }^{\omega} 2$, define $\mathscr{A}(X)=\{a \subset S q \cup X: a$ is a finite nonmaximal antichain in the binary tree $\left.{ }^{<\omega+1} 2\right\} . \mathscr{A}(X)$ is ordered by reverse inclusion.

We shall refer to the ground model (before adding the Sacks reals) by $V$ and use $V[G]$ to denote the extension. Fix, in $V$, an indexing $\left\{a_{m}: m<\omega\right\}$ of $\mathscr{A}(0)$. For any $a \in \mathscr{A}(0)$, let $[a]=\bigcup\{[s]: s \in a\}$ and note that $[a]$ is a clopen subset of $C$. For any $X \subset C$ and $a \in \mathscr{A}(X)$ let $[a]=[a-X]$. Observe that for any $x \in X$ and $a \in \mathscr{A}(X), a \cup\{x\} \in \mathscr{A}(X)$ iff $x \notin[a]$.

Proposition 5.2. For any $X \subset C, \mathscr{A}(X)$ is $\sigma$-centered.

Proof. Indeed by the above remark, for each $m \in \omega$, the collection $\{a \in$ $\left.\mathscr{A}(X): a-X=a_{m}\right\}$ is centered.

The posets $\mathscr{A}(X)$ for various $X \subset C$ are also investigated in [D5] and the following is proved there.

Proposition 5.3. (a) For any $n \in \omega, \mathscr{A}(C)$ has a remote $n$-linked collection.

(b) If $X \subset C$ is such that $|X|>\omega, x+q \in X$ for each $x \in X$ and dyadic rational $q \in C$, and $X$ contains no perfect subset then $\mathscr{A}(X)$ has no remote 2-linked collection.

Although we do not require 5.3(a) here, it will be useful to sketch the ideas in the proof of 5.3(b) since a more general version is required for our main result.

The basic idea of the property of $X$ in (b) is that any uncountable subset of $X$ must have a limit point in $C-X$. The additional restriction (that $X$ is closed under translates by rationals) is a device which allows us to show that any uncountably many specially chosen $n$-tuples in $X^{n}$ will have limit points in $(C-X)^{n}$. Finally Proposition 4.3 is designed to guarantee this same phenomenon will occur with countable sequences in $\square C$.

Indeed suppose that $\mathscr{L}$ is a remote collection on $\mathscr{A}(X)$ (i.e. $\left.\mathscr{L} \subset[\mathscr{A}(X)]^{<\omega}\right)$. Let us first suppose that $\mathscr{L} \cap[\mathscr{A}(X)]^{1}$ is uncountable. Then $\{x \in X: \exists a \in$ $\mathscr{A}(X)$ with $x \in a, a \in \mathscr{L}\}$ is uncountable, hence there is some $y \in C-X$ which is a limit point of this set. Also since $y \notin X$ the collection $\{\{y \mid n\}: 0<$ $n<\omega\}$ is predense in $\mathscr{A}(X)$. It follows without loss of generality that, for some $n<\omega$, the collection $\{\{y \mid m\}: 0<m<n\} \in \mathscr{L}$. Now since $y$ is a limit point of the above set, there is an $x \in[y \mid n]$ and an $\{a\} \in \mathscr{L}$ with $x \in a$. Therefore $\mathscr{L}$ is not 2-linked since $\{y \mid m\} \cup a \notin \mathscr{A}(X)$ for any $m<n$. More generally, we can find an $n<\omega$ so that there are $\omega_{1}$ distinct sequences $\left\langle x_{0}^{\alpha}, \ldots, x_{n-1}^{\alpha}\right\rangle \in X^{n}$ such that $\left\{a_{m} \cup\left\{x_{m}^{(\alpha)}\right\}: m<n\right\} \in \mathscr{L}$ for $\alpha<\omega_{1}$. We then consider $\left\langle y_{0}, \ldots, y_{n-1}\right\rangle \in(C-X)^{n}$ such that $\left\langle y_{0}, \ldots, y_{n-1}\right\rangle$ is a limit point of $\left\{\left\langle x_{0}^{\alpha}, \ldots, x_{n-1}^{\alpha}\right\rangle: \alpha<\omega_{1}\right\}$. In a similar fashion we show that the predense set $\left\{a \in \mathscr{A}(X):\left\{y_{0}, \ldots, y_{n-1}\right\} \subset[a]\right\}$ cannot have a finite subset in $\mathscr{L}$ making $\mathscr{L}$ 2-linked.

Now let us prove the main theorem. 
Theorem 5.4. For $\alpha \in \omega_{2}$, let $x_{\alpha} \in C$ be as in $\S 3$ and let $X=\left\{x_{\alpha}: \omega_{1} \leq\right.$ $\left.\alpha<\omega_{2}\right\}$. Then $\sum_{\omega} \mathscr{A}(X)$ has no remote filters (in fact, no remote 2 -linked collection).

Proof. Let $g$ be any injection (in $V$ ) of $\omega \times \omega$ into $\omega$. Fix $\left\{f_{\alpha}: \alpha<\omega_{1}\right\} \subset$ ${ }^{\omega} \omega \cap V$ such that for all $f \in{ }^{\omega} \omega \cap V[G]$ there is an $\alpha<\omega_{1}$ with $f(n)<f_{\alpha}(n)$ for all $n$ (by 3.3). In $V[G]$, let $J=\left\{\lambda \in \omega_{2}-\omega_{1}: a_{m} \cup\left\{x_{\lambda+g(n, m)}\right\} \in \mathscr{A}(X)\right.$ for each $n, m \in \omega\}$. We claim that $|J|=\omega_{2}$. Indeed, for each $n, m \in \omega$, we may choose $s_{g(n, \omega)} \in S q$ such that $a_{m} \cup\left\{s_{g(n, m)}\right\} \in \mathscr{A}(0)$ and the function taking $(n, m)$ to $s_{g(n, m)}$ for $n, m \in \omega$ is in $V$. Clearly $J \supset\left\{\lambda \in \omega_{2}-\omega_{1}: x_{\lambda+g(n, m)} \supset\right.$ $s_{g(n, m)}$ for each $\left.n, m \in \omega\right\}$, hence $|J|=\omega_{2}$ by 4.3 .

For each $\lambda \in J$ and $n, m \in \omega$, let $A(\lambda, n)=\left\{a_{m} \cup\left\{x_{\lambda+g(n, m)}\right\}: m \in \omega\right\}$. Clearly $A(\lambda, n)$ is predense in $\mathscr{A}(X)$, hence $\bigcup\{\{n\} \times A(\lambda, n): n<\omega\}$ is predense in $\sum_{\omega} \mathscr{A}(X)$. Suppose $\mathscr{F}$ is a remote collection on $\sum_{\omega} \mathscr{A}(X)$. Then for each $\lambda \in J$, there is an $\alpha<\omega_{1}$ such that $\bigcup\left\{\{n\} \times\left\{a_{m} \cup\left\{x_{\lambda+g(n, m)}\right\}: m<\right.\right.$ $\left.f_{\alpha}(n)\right\} \in \mathscr{F}$ (we may assume $\mathscr{F}$ is 'closed upwards'). Therefore there are an $\alpha<\omega_{1}$ and a $J_{0} \subset J$ with $\left|J_{0}\right|=\omega_{2}$ such that for all $\lambda \in J_{0} \bigcup\left\{\{n\} \times\left\{a_{m} \cup\right.\right.$ $\left.\left.\left\{x_{\lambda+g(n, m)}\right\}: m<f_{\alpha}(n)\right\}: n<\omega\right\} \in \mathscr{F}$. For each $\lambda \in \lim \left(\omega_{2}\right)$, define $y_{\lambda} \in$ $\prod_{n \in \omega} C^{f_{n}(n)}$ by $y_{\lambda}(n)=\left\langle x_{\lambda+g(n, 0)}, \ldots, x_{\lambda+g\left(n, f_{n}(n)-1\right.}\right\rangle$. Let $Z=\left\{y_{\lambda}: \lambda \in J_{0}\right\}$ and consider $Z$ as a subspace of $\prod_{n \in \omega} C^{f_{n}(n)}$ with the box topology, which is clearly homeomorphic to $\square C$. By 4.3, there is a $\delta \in \lim \left(\omega_{1}\right)$, such that $y_{\delta}$ is a limit point of $Z$ (in the box topology). For each $n \in \omega$, define $A_{n} \subset \mathscr{A}(X)$ by

$$
A_{n}=\left\{a \in \mathscr{A}(X):[a] \supset\left\{x_{\delta+g(n, m)}: m<f_{\alpha}(n)\right\}\right\} .
$$

Let us check that $A_{n}$ is predense. Indeed suppose $a^{\prime} \in \mathscr{A}(X)$ and $m<f_{\alpha}(n)$ is the minimum such that $x_{\delta+g(n, m)} \notin\left[a^{\prime}\right]$. Since $x_{\delta+g(n, m)} \notin X$ there is a $k<\omega$ such that $\left[x_{\delta+g(n, m)} \mid k\right] \cap\left(\left[a^{\prime}\right] \cup\left(a^{\prime} \cap X\right)\right)=0$. It follows that $a^{\prime} \cup\left\{x_{\delta+g(n, m)} \mid k\right\} \in \mathscr{A}(X)$. Hence, by induction, $A_{n}$ is predense.

Now, suppose $A_{n}^{\prime} \in\left[A_{n}\right]^{<\omega}$ for $n \in \omega$ are such that $\bigcup\left\{\{n\} \times A_{n}^{\prime}: n<\right.$ $\omega\} \in \mathscr{F}$. For each $n<\omega$, let $U_{n}=\bigcap\left\{[a]: a \in A_{n}^{\prime}\right\}$ and consider the box neighborhood $W=\prod_{n \in \omega} U_{n}^{f_{n}(n)}$ of $y_{\delta}$. By assumption on $y_{\delta}$ there is a $\lambda \in J_{0}$ such that $y_{\lambda} \in W$. Finally, for each $n \in \omega, a \in A_{n}^{\prime}$, and $m<f_{\alpha}(n)$, $a \cup\left\{x_{\lambda+g(n, m)}\right\} \notin \mathscr{A}(X)$ (since $x_{\lambda+g(n, m)} \in[a]$ ) hence $\mathscr{F}^{n}$ is not 2-linked.

\section{NECESSARY CONDITIONS FOR REMOTE POINTS IN $v X$}

As mentioned in the introduction Terada has shown that if $X$ has remote points in $v X$ then the cellularity of $X$ must be at least as large as the first measurable cardinal, $\mu_{0}$. This was improved by Blair who proved that there must also be a closed discrete set of cardinality $\mu_{0}$ which can be separated by disjoint open sets. In this section we shall improve these results to obtain a much more complete picture of which spaces $X$ do not have remote points 
in $v X$. For instance we show that if $X$ has remote points in $v X$ then $X$ is not pseudo- $\mu_{0}$-compact (recall that $X$ is pseudo- $\lambda$-compact if each locally finite family of open sets has cardinality less than $\lambda$ ). An immediate corollary is that a pseudocompact space does not have remote points (since $v X=\beta X$ ) (Terada showed this for $X$ with cellularity less than $\mu_{0}$ ).

For the remainder of this section, let $X$ be a space and let $p \in v X-X$ be a remote point of $X$. We shall derive some properties of $X$ in relation to the ultrafilter $p$.

Lemma 6.1. For each $Z \in p, p \in$ int $_{\beta X} \operatorname{cl}_{\beta X} Z$.

Proof. The set $D=Z \cap \mathrm{cl}_{X}(X-Z)$ is a nowhere dense subset of $X$, hence $p \notin \mathrm{cl}_{\beta X} D$. Choose $h \in C^{*}(\beta X)$ such that $h(p)=0$ and $D \subset$ int $_{\beta X} h^{-1}(1)$. Define $h_{0} \in C^{*}(X)$ as follows: $h_{0}(x)=h(x)$ for $x \in Z$ and $h_{0}(x)=1$ for $x \in X-Z$. Since $h$ is identically 1 on a neighborhood of $D, h_{0}$ is continuous. Now clearly $\beta h_{0}^{-1}((-\infty, 1 / 2))$ is a $\beta X$-neighborhood of $p$ entirely contained in $\mathrm{cl}_{\beta X} Z$.

Recall that $p$ is a $P_{\kappa}$-point $\left(P_{\omega_{1}}\right.$-point $=P$-point $)$ of $\beta X$ if $p \in$ int $\cap \mathscr{U}$ for any family $\mathscr{U}$ of fewer than $\kappa$ many neighborhoods of $p$.

Corollary 6.2. $p$ is a P-point of $\beta X$.

Proof. Since $p \in v X-X$, as a zero-set ultrafilter, $p$ is $\omega_{1}$-complete. Therefore by $6.1, p$ is a $P$-point.

The next result is really the main result of this section and it shows that, as far as the point $p$ is concerned, the space $X$ is really just a free union of an infinite collection of cozero sets.

Lemma 6.3. For any maximal pairwise disjoint family $\mathscr{A}$ of cozero subsets of $X$ such that $p \notin \mathrm{cl}_{\beta X} A$ for $A \in \mathscr{A}$, there is a zero set $Z \in p$ such that the family $\{A \cap Z: A \in \mathscr{A}\}$ is locally finite, $Z=\bigcup\{A \cap Z: A \in \mathscr{A}\}$ and $A \cap Z \in \mathscr{Z}(X)$ for each $A \in \mathscr{A}$.

Proof. Since $p \notin \operatorname{cl}_{\beta X}(X-\bigcup \mathscr{A}$ ) (by maximality of $\mathscr{A}$ ), we may choose $f: \beta X \rightarrow[0,1]$ such that $p \in \operatorname{int}_{\beta X} f^{-1}(0)$ and $X-\cup \mathscr{A} \subset \operatorname{int}_{\beta X} f^{-1}(1)$. Let $Z=f^{-1}(0) \cap X$. For $A \in \mathscr{A}$, choose $g_{A}: X \rightarrow[0,1]$ such that $A=X-g_{A}^{-1}(0)$ and define $h_{A}$ to be identically 0 on $X-A$ and equal to $g_{A} /\left(g_{A}+f \uparrow A\right)$ on $A$. Again, $h_{A} \in C^{*}(X)$ since $f$ is identically 1 on the boundary of $A$ and $A \cap Z=h_{A}^{-1}(1) \in \mathscr{Z}(X)$. The family $\mathscr{A}$ exhibits the local finiteness of $\{A \cap Z: A \in \mathscr{A}\}$ since $\mathrm{cl}_{X} Z=Z \subset \bigcup \mathscr{A}$.

Corollary 6.4. $X$ is not pseudocompact.

To further explore the properties of $X$ in relation to $p$ we introduce some definitions. For any space $Y$, define $\operatorname{wn}(Y)$ (the weak Novak number) to be $\min \{|\mathscr{D}|: \mathscr{D}$ is a family of nowhere dense sets whose union is dense $\}$ (if $Y$ has isolated points, let $\mathrm{wn}(Y)=\infty$ where $\kappa<\infty$ for all cardinals $\kappa)$. Recall that $\pi w(Y)=\min \{|\mathscr{B}|: \mathscr{B}$ is a $\pi$-base for $Y\}$ (a collection $\mathscr{B}$ of nonempty open 
subsets of $Y$ is a $\pi$-base for $Y$ if for each nonempty open $U \subset Y$ there is a $B \in \mathscr{B}$ with $B \subset U)$.

Fix a maximal pairwise disjoint family $\mathscr{A}^{\prime}$ of cozero sets so that $p \notin \mathrm{cl}_{\beta X} A$ for $A \in \mathscr{A}^{\prime}$. Further, suppose that $\mathrm{wn}(U)=\mathrm{wn}(A)$ and $\pi w(U)=\pi w(A)$ for each $A \in \mathscr{A}^{\prime}$ and nonempty open $U \subset A$ (this is no loss of generality since $\mathrm{wn}(U) \leq \mathrm{wn}(A)$ and $\pi w(U) \leq \pi w(A)$ for each nonempty open $U \subset A$ for any open $A)$. Let $\mathscr{A} \subset \mathscr{A}^{\prime}$ be of minimum cardinality such that $p \in \mathrm{cl}_{\beta X} \cup \mathscr{A}$; let $\lambda=|\mathscr{A}|$ and fix an indexing $\left\{A_{\alpha}: \alpha<\lambda\right\}=\mathscr{A}$. Let us observe the following.

Lemma 6.5. Let $Z \in p$ be chosen as in 6.3 for the family $\mathscr{A}^{\prime}$. For any $\mathscr{V} \subset$ $\left\{A \cap Z: A \in \mathscr{A}^{\prime}\right\}, \bigcup \mathscr{V} \in \mathscr{Z}(X)$.

Proof. Let $f$ be chosen as in 6.3 such that $Z=f^{-1}(0) \cap X$ and int $_{\beta X} f^{-1}(1) \supset$ $X-\bigcup \mathscr{A}^{\prime}$. Let $\hat{h}_{A}=h_{A} \cdot(1-f)$ where $h_{A}$ is as in 6.3. It follows now that $A \cap Z=\hat{h}_{A}^{-1}(1)=h_{A}^{-1}(1)$ and the family $\left\{X-\hat{h}_{A}^{-1}(0)\left(=A-h_{A}^{-1}(0)\right): A \in \mathscr{A}^{\prime}\right\}$ is locally finite. Therefore the function $h_{\mathscr{V}}=\sum\left\{\hat{h}_{A}: A \in \mathscr{A}^{\prime}\right.$ and $A \cap Z \in$ $\mathscr{V}\} \in C^{*}(X)$ and $h_{\mathscr{V}}^{-1}(1)=\bigcup \mathscr{V} \in \mathscr{Z}(X)$.

Note that by 6.5 and 6.1, $p \notin \operatorname{cl}_{\beta X}(X)-\bigcup\{A \cap Z: A \in \mathscr{A}\}$. Furthermore, let $I(X)$ be the isolated points of $X$ and assume without loss of generality that $\{\{x\}: x \in I(X)\} \subset \mathscr{A}^{\prime}$. By 6.5, either $p$ is essentially a member of $\beta(I(X))$ or $p \in \beta X-\operatorname{cl}_{\beta X} I(X)$ and $I(X)$ has no effect on $p$. This is why we assumed in the introduction that $X$ has no isolated points rather than simply that $p \in \mathrm{cl}_{\beta X}(X-I(X))$.

Definition 6.6. (i) Let $\kappa_{p}=\min \{|\mathscr{Z}|: \mathscr{Z} \subset p \cap \mathscr{Z}(X)$ and $\cap \mathscr{Z}=0\}$.

(ii) Let $\mathscr{U}_{p} \subset P(\lambda)$ be defined by $I \in \mathscr{U}_{p}$ iff $p \in \operatorname{cl}_{\beta X} \cup\left\{A_{\alpha}: \alpha \in I\right\}$, and let $\lambda_{p}=\lambda$.

(iii) Let $\gamma_{p}=\min \left\{|\mathscr{U}|: \mathscr{U} \subset \mathscr{U}_{p}\right.$ and $\left.\cap \mathscr{U}=0\right\}$.

(iv) Let $w_{p}=\inf \left\{\sup \left\{\operatorname{wn}\left(A_{\alpha}\right): \alpha \in I\right\}: I \in \mathscr{U}_{p}\right\}$.

We will now show that $\mu_{0} \leq \min \left\{\kappa_{p}, \lambda_{p}, \gamma_{p}, w_{p}\right\}$. Let us begin with what is essentially Terada's result.

Proposition 6.7. $\mathscr{U}_{p}$ is an ultrafilter on $\lambda_{p}$ and $\kappa_{p} \leq \gamma_{p}$ and $\gamma_{p}$ is measurable. Proof. That $\mathscr{U}_{p}$ is an ultrafilter follows from 6.5 and the fact that disjoint zero sets have disjoint closures in $\beta X$. Furthermore 6.5 implies that $\kappa_{p} \leq \gamma_{p}$. Indeed let $\left\{I_{\beta}: \beta<\gamma_{p}\right\} \subset \mathscr{U}_{p}$ be such that $\bigcap\left\{I_{\beta}: \beta<\gamma_{p}\right\}=0$. Then for each $\beta<\gamma_{p}, Z_{\beta}=\bigcup\left\{A_{\alpha} \cap Z: \alpha \in I_{\beta}\right\} \in p$ and $\left\{Z_{\beta}: \beta<\gamma_{p}\right\}=0$. Finally, by 6.2, $\omega<\kappa_{p} \leq \gamma_{p}$ hence $\gamma_{p}$ is measurable by the definition of a measurable cardinal.

Theorem 6.8. $p$ is a $P_{\kappa_{p}}$-point of $\beta X$ and $\kappa_{p}$ is measurable.

Proof. Suppose $\mathscr{Z},|\mathscr{Z}|<\kappa_{p}$, is a collection of zero set neighborhoods in $\beta X$ of $p$. Let $U=$ int $_{X} \cap \mathscr{Z}$ and $D=X-U \cap \cap \mathscr{Z}$. Since $D$ is nowhere dense in $X$, we may choose $W \in p$ with $W \cap D=0$. Since $|\mathscr{Z}|<\kappa_{p}$, 
$W \cap U=\bigcap\{W \cap Z: Z \in \mathscr{Z}\} \neq 0$ and it follows that $p \in \operatorname{cl}_{\beta X} U$. To prove that $p$ is a $P_{\kappa_{p}}$-point of $\beta X$ it suffices to show that $p \notin \mathrm{cl}_{\beta X}(X-U)$, since this implies $p \in \operatorname{int}_{\beta X} \mathrm{cl}_{\beta X} U \subset \cap \mathscr{Z}$. This fact follows from results in [vD2] or by choosing $\mathscr{A}$ as in 6.3 to refine $\{U, X-\bar{U}\}$ and applying 6.5. To show that $\kappa_{p}$ is measurable we show there is a $\kappa_{p}$-complete ultrafilter on $\kappa_{p}$. Choose $\left\{Z_{\alpha}: \alpha<\kappa_{p}\right\} \subset p$ so that $W_{\alpha} \subset \operatorname{int}_{\beta X} Z_{\alpha}^{p} \cap \cap\left\{\operatorname{cl}_{\beta X}\left(W_{\beta} \cap Z_{\beta}\right): \beta<\alpha\right\}$, which we may do by 6.1 and the above. Let, for $\alpha<\kappa_{p}, A_{\alpha}^{\prime}=\operatorname{int}_{X} \cap\left\{W_{\beta}-W_{\alpha}: \beta<\alpha\right\}$ with $A_{0}^{\prime}=X-W_{0}$. Choose any $f \in C^{*}(\beta X)$ such that $f(p)=0$ and $f^{-1}(1) \supset$ $X-\bigcup\left\{A_{\alpha}^{\prime}: \alpha<\kappa_{p}\right\}$. For each $\alpha<\kappa_{p}$, let $A_{\alpha}=A_{\alpha}^{\prime} \cap f^{-1}((-1 / 2,1 / 2))$. It follows that $p \in \operatorname{cl}_{\beta X} \bigcup\left\{A_{\alpha}: \alpha<\kappa_{p}\right\}$ and each $A_{\alpha}$ is a cozero set in $X$ (i.e. just use the function $|f|$ on $A_{\alpha}$ and constant $1 / 2$ on $X-A_{\alpha}$ ). Now apply 6.3 and 6.7 with $\lambda_{p}=\kappa_{p}$ and a new $\mathscr{U}_{p}$.

Perhaps the most surprising and strongest result that we have is the following.

Theorem 6.9. $\kappa_{p} \leq w_{p}$, hence $\mu_{0} \leq w_{p}$.

Proof. Choose $U \in \mathscr{U}_{p}$ such that $\sup \left\{\operatorname{wn}\left(A_{\alpha}\right): \alpha \in U\right\}=w_{p}$. We may as well assume $w_{p}<\infty$. For each $\alpha \in U$, fix $\mathscr{D}_{\alpha}=\left\{D_{\gamma}^{\alpha}: \gamma<w_{p}\right\}$ exhibiting that wn $\left(A_{\alpha}\right) \leq w_{p}$. Define, for $\gamma<w_{p}, D_{\gamma}=\bigcup\left\{D_{\gamma}^{\alpha}: \alpha \in U\right\}$ and observe that $D_{\gamma}$ is a nowhere dense subset of $X$ (and $\beta X$ ). Furthermore, $\operatorname{cl}_{\beta X} \bigcup\left\{D_{\gamma}: \gamma<w_{p}\right\}=$ $\mathrm{cl}_{\beta X} \bigcup\left\{A_{\alpha}: \alpha \in U\right\}$ from which it follows that $p \notin \operatorname{int}_{\beta X} \cap\left\{\beta X-D_{\gamma}: \gamma<w_{p}\right\}$. Since $p \in$ int $_{\beta X} \beta X-D_{\gamma}$ for each $\gamma<w_{p}$, it follows, from 6.8, that $\kappa_{p} \leq w_{p}$.

The upshot of 6.9, which is new, is that if $X$ has remote points in $v X$ then $X$ is not constructed as a free union (however large) of small spaces. For example let us state the following corollary.

Corollary 6.10. The space $X=\lambda \times R$ does not have remote points in $v X$ for any $\lambda$.

Remark 6.11. Clearly the definition of $\lambda_{p}, \gamma_{p}$ and $w_{p}$ depend on $\mathscr{A}$ (making the restriction of "homogeneous" $\pi$-weight and weak Novak number for $A \in \mathscr{A}$ was just an attempt to make $\mathscr{A}$ canonical). In fact with these restrictions on $\mathscr{A}, w_{p}$ does not depend on $\mathscr{A}$ and we could define $\lambda_{p}$ to be minimum possible with respect to such homogeneous $\mathscr{A}$. We have shown that $\kappa_{p} \leq \gamma_{p} \leq \lambda_{p}$ and $\kappa_{p} \leq w_{p}$. In the next section we shall see that these inequalities can be strict. Furthermore we can have $w_{p}<\gamma_{p}$ or $\gamma_{p}<w_{p}$, but I do not know if $\lambda_{p}<w_{p}$ is possible.

\section{SOME EXAMPLES WITH REMOTE POINTS IN $v X$}

In order to produce an example of a space $X$ with a remote point in $v X$ we shall obviously have to assume the existence of measurable cardinals. In fact we shall work with a supercompact cardinal.

Definition 7.1. A cardinal $\kappa$ is supercompact if for each $\lambda, \lambda \geq \kappa$, there is a $\kappa$-complete normal fine ultrafilter $\mathscr{U}$ on $[\lambda]^{<\kappa}$. (Recall that $[\lambda]^{<\kappa}=\{a \subset$ $\lambda:|a|<\kappa\} ; \mathscr{U}$ is called normal if for each regressive function $f$ on a member 
of $\mathscr{U}$ (i.e. $f(a) \in a$ for all $a \in U$ for some $U \in \mathscr{U}$ ) there is an $\alpha \in \lambda$ such that $f^{-1}(\alpha) \in \mathscr{U} ; \mathscr{U}$ is fine if $\langle\alpha\rangle=\left\{a \in[\lambda]^{<\kappa}: \alpha \in a\right\} \in \mathscr{U}$ for all $\left.\alpha \in \lambda\right)$.

Facts 7.2 [KaMa]. (a) If $\kappa$ is measurable then there is a $\kappa$-complete normal fine ultrafilter on $[\kappa]^{<\kappa}$.

(b) If $\kappa$ is supercompact and $\lambda^{<\kappa}=\lambda$ then $|U|=\lambda$ for each $U \in \mathscr{U}$ where $\mathscr{U}$ is as in 7.1.

We shall show, for certain spaces $X$ of the form $\sum\left\{X_{a}: a \in[\lambda]^{<\kappa}\right\}$, where $\kappa$ is measurable, that $X$ has a remote point $p$ in $v X$ with $\kappa_{p}=\kappa$. We know from $\S 6$ that it is necessary that $w_{p} \geq \kappa$, however for our induction to work we will actually require a stronger completeness property on $X$. Recall that a poset is said to be $\lambda$ directed closed if every directed subset of size less than $\lambda$ has a lower bound. For a space $Y$ let $G(Y)$ be the supremum of all $\lambda$ such that $Y$ has a $\pi$-base which is $\lambda$ directed closed when ordered by inclusion. The relationship between $G(Y)$ and $\mathrm{wn}(Y)$ is that $G(Y) \leq \mathrm{wn}(U)$ for all nonempty open $U \subset Y$.

Theorem 7.3. Let $\kappa \leq \lambda$ be cardinals such that $2^{\lambda}=\lambda^{+}$and there is a $\kappa$ complete normal fine ultrafilter $\mathscr{U}$ on $[\lambda]^{<\kappa}$. Let $\left\{X_{a}: a \in[\lambda]^{<\kappa}\right\}$ be a family of spaces such that $\pi w\left(X_{a}\right) \leq \lambda^{+}, c\left(X_{a}\right) \leq \lambda^{+}$and $G\left(X_{a}\right)>|a|$ for all $a \in[\lambda]^{<\kappa}$. Then the space $X=\sum\left\{X_{a}: a \in[\lambda]^{<\kappa}\right\}$ has a remote point $p \in v X$ such that $\mathscr{U}_{p}=\mathscr{U}$ where $\mathscr{U}_{p}$ and $\mathscr{A}=\left\{X_{a}: a \in[\lambda]^{<\kappa}\right\}$ are as in 6.3 and 6.6 .

Proof. For each $a \in[\lambda]^{<\kappa}$, fix an $|a|^{+}$directed closed $\pi$-base $\mathscr{B}_{a}$ for $X_{a}$. Since $\pi w\left(X_{a}\right) \leq \lambda^{+}$and $2^{\lambda}=\lambda^{+}$we may suppose that $\left|\mathscr{B}_{a}\right| \leq \lambda^{+}$for each $a \in$ $[\lambda]^{<\kappa}$ (i.e. choose a $\pi$-base $\mathscr{B}_{a}^{\prime}$ of size at most $\lambda^{+}$and choose an $|a|^{+}$directed closed subset of $\mathscr{B}_{a}$ which refines $\mathscr{B}_{a}^{\prime}$ ). Under our current assumptions $X$ has cellularity $\lambda^{+}$hence the family of all maximal pairwise disjoint subsets of $\bigcup\left\{\mathscr{B}_{a}: a \in[\lambda]^{<\kappa}\right\}$ has cardinality $\left(\lambda^{+}\right)^{\lambda}=\lambda^{+}$. Let $\left\{\mathscr{C}_{\xi}: \xi<\lambda^{+}\right\}$index this family. Let $\xi<\lambda^{+}$and suppose that for $\gamma<\xi$ we have chosen $f_{\gamma}$ a function from $[\lambda]^{<\kappa}$ into $\bigcup\left\{\mathscr{B}_{a}: a \in[\lambda]^{<\kappa}\right\}$ so that

(i) for each $a \in[\lambda]^{<\kappa} f_{\gamma}(a) \in \mathscr{B}_{a}$ and there is a $B \in \mathscr{C}_{\gamma}$ such that $f_{\gamma}(a) \subset B$ and $f_{\gamma}(a)$ is completely separated from $X_{a}-B$, and

(ii) for any $F \subset X$ which is a finite intersection of members of the family $\left\{\bigcup\left\{f_{\gamma}(a): a \in[\lambda]^{<\kappa}\right\}: \gamma<\xi\right\},\left\{a \in[\lambda]^{<\kappa}: F \cap X_{a} \neq 0\right\} \in \mathscr{U}$.

Let us choose $f_{\xi}$. Let $\left\{F_{\alpha}: \alpha<\lambda\right\}$ list all finite intersections from the family $\left\{\bigcup\left\{f_{\gamma}(a): a \in[\lambda]^{<\kappa}\right\}: \gamma<\xi\right\}$. Let us show that the set $U=\left\{a \in[\lambda]^{<\kappa}:\left\{F_{\alpha} \cap\right.\right.$ $\left.X_{a}: \alpha \in a\right\}$ is directed $\}$ is in $\mathscr{U}$. Suppose not and for each $a \in[\lambda]^{<\kappa}-U$ choose $m_{a} \in \omega$ and $\left\{\alpha_{n}(a): n<m_{a}\right\} \subset a$ so that $\bigcap\left\{F_{\alpha_{n}(a)} \cap X_{a}: n<m_{a}\right\}=0$. By the $\kappa$-completeness of $\mathscr{U}$ there is an $m<\omega$ and a $V \in \mathscr{U}$ such that $m_{a}=m$ for all $a \in V$. Next, by the normality of $\mathscr{U}$ used $m$ times, there is a $V^{\prime} \in \mathscr{U}$ and a sequence $\left\{\alpha_{n}: n<m\right\} \subset \lambda$ such that $\alpha_{n}(a)=\alpha_{n}$ for each $n<m$ and $a \in V^{\prime}$. However, by (ii), $W=\left\{a \in[\lambda]^{<\kappa}: X_{a} \cap \cap\left\{F_{\alpha_{n}}: n<m\right\} \neq 0\right\} \in \mathscr{U}$ 
and $V \cap V^{\prime} \cap W=0$. Therefore $U \in \mathscr{U}$. For $a \notin U$, choose $f_{\xi}(a) \in \mathscr{B}_{a}$ so as to satisfy (i). For $a \in U$, we have that $\bigcap\left\{F_{\alpha} \cap X_{a}: \alpha \in a\right\}$ has nonempty interior since $\mathscr{B}_{a}$ is $|a|^{+}$directed closed, $\left\{F_{\alpha} \cap X_{a}: \alpha \in a\right\}$ is directed and each $F_{\alpha} \cap X_{a}$ is a finite intersection of members of $\mathscr{B}_{a}$. Since $\mathscr{C}_{\xi}$ is a maximal almost disjoint family of open sets we may choose $f_{\xi}(a) \in \mathscr{B} a$ so that (i) holds and $f_{\xi}(a) \subset \bigcap\left\{F_{\alpha} \cap X_{a}: \alpha \in a\right\}$. To see that (ii) holds note that for $\alpha<\lambda\left\{a \in[\lambda]^{<\kappa}: F_{\alpha} \cap f_{\xi}(a) \neq 0\right\} \supset\langle\alpha\rangle \cap U \in \mathscr{U}$.

Let $\mathscr{F} \subset \mathscr{Z}(X)$ be the filter generated by those $Z \in \mathscr{Z}(X)$ such that for some $U \in \mathscr{U}$ and $\xi<\lambda^{+} \cup\left\{f_{\xi}(a): a \in U\right\} \subset Z$ (that $\mathscr{F}$ is a filter follows from property (ii)). Note that for each $\xi<\lambda^{+}$there is a $Z_{\xi} \in \mathscr{Z}(X)$ and a cozero set $C_{\xi}$ of $X$ such that for each $a \in[\lambda]^{<\kappa}$ there is a unique $B \in \mathscr{C}_{\xi}$ such that $f_{\xi}(a) \subset Z_{\xi} \cap X_{a} \subset C_{\xi} \cap X_{a} \subset B$ (by property (i)). Furthermore observe that from the proof that (ii) holds in the induction step above it follows easily that for any $\left\{\xi_{n}: n<\omega\right\} \subset \lambda^{+},\left\{a \in[\lambda]^{<\kappa}:\left\{f_{\xi_{n}}(a): n<\omega\right\}\right.$ is directed $\} \in \mathscr{U}$. Now let us show that $\mathscr{F}$ is a remote ultrafilter and that $\mathscr{F} \in v X$. For any nowhere dense set $D \subset X$, there is a $\xi<\lambda^{+}$such that $\bigcup \mathscr{C}_{\xi} \cap D=0$. Since $Z_{\xi} \subset C_{\xi}$ and $D \subset X-C_{\xi}, Z_{\xi}$ is completely separated from $D$, hence $\mathscr{F}$ is remote. To see that $\mathscr{F}$ is an ultrafilter, let $W \in \mathscr{Z}(X)$. Choose $\xi<\lambda^{+}$such that $\mathscr{C}_{\xi}$ refines $\{$ int $W, X-W\}$. Therefore, for each $a \in[\lambda]^{<\kappa}$ either $Z_{\xi} \cap X_{a} \subset W$ or $Z_{\xi} \cap X_{a} \cap W=0$. If $U=\left\{a \in[\lambda]^{<\kappa}: Z_{\xi} \cap X_{a} \subset W\right\} \in \mathscr{U}$ then $W \in \mathscr{F}$ while if $U \in \mathscr{F}$ then $Z_{\xi}^{\prime}=Z_{\xi} \cap \sum\left\{X_{a}: a \notin U\right\} \in \mathscr{F}$ and $Z_{\xi}^{\prime} \cap W=0$. Note that in fact for each $Z \in \mathscr{F}$ there is a $\xi<\lambda^{+}$and a $U \in \mathscr{U}$ such that $\bigcup\left\{f_{\xi}(a): a \in U\right\} \subset Z$. Now to show that $\mathscr{F} \in v X$ it suffices to show that $\bigcap\left\{Z_{n}: n<\omega\right\} \neq 0$ for any $\left\{Z_{n}: n<\omega\right\} \subset \mathscr{F}$. So let $\left\{Z_{n}: n<\omega\right\} \subset \mathscr{F}$ and choose $\left\{\xi_{n}: n<\omega\right\} \subset \lambda^{+}$and $\left\{U_{n}: n<\omega\right\} \subset \mathscr{U}$ so that $\bigcup\left\{f_{\xi_{n}}(a): a \in U_{n}\right\} \subset$ $Z_{n}$. Let $U=\bigcap\left\{U_{n}: n<\omega\right\} \cap\left\{a \in[\lambda]^{<\omega}:\left\{f_{\xi_{n}}(a): n<\omega\right\}\right.$ is directed $\} \in \mathscr{U}$. Therefore for any infinite $a \in U, \bigcap\left\{Z_{n} \cap X_{a}: n<\omega\right\} \supset \bigcap\left\{f_{\xi_{n}}(a): n<\omega\right\} \neq 0$ since $\left\{f_{\xi_{n}}(a): n<\omega\right\}$ is a directed subset of the $|a|^{+}$-directed closed $\pi$-base $\mathscr{B}_{a}$.

To apply 7.3 we need a class of spaces satisfying the hypotheses. For a cardinal $\alpha$ let $\left(2^{\alpha}\right)_{\alpha}$ be the refinement of the usual topology on $2^{\alpha}$ which has $\left\{[s]: s \in{ }^{<\alpha} 2\right\}$ as a base where $[s]=\left\{f \in 2^{\alpha}: s \subset f\right\}$ for $s \in{ }^{<\alpha} 2=\left\{{ }^{\beta} 2: \beta<\alpha\right\}$. Let $Y_{\alpha}$ be the Stone-Čech compactification of $\left(2^{\alpha}\right)_{\alpha}$. If $\alpha$ is regular then $\pi w\left(Y_{\alpha}\right)=\pi w\left(\left(2^{\alpha}\right)_{\alpha}\right)=2^{<\alpha}, G\left(Y_{\alpha}\right)=G\left(\left(2^{\alpha}\right)_{\alpha}\right)=\alpha$ and $c\left(Y_{\alpha}\right)=c\left(\left(2^{\alpha}\right)_{\alpha}\right)=$ $2^{<\alpha}$.

Let us now list some examples.

Example 7.4. If $\kappa$ is measurable and $2^{\kappa}=\kappa^{+}$then there is a space $X$ with no isolated points which has a remote point $p$ in $v X$. We can also have $X=\sum\left\{X_{\alpha}: \alpha \in \kappa\right\}$ such that $\operatorname{wn}\left(X_{\alpha x}\right)<\kappa$ for all $\alpha<\kappa$. Indeed, let $\mathscr{U}$ be a normal $\kappa$-complete ultrafilter on $\kappa$ (think of $\kappa$ as a subset of $[\kappa]^{<\kappa}$ ) and for each $\alpha<\kappa$ let $X_{\alpha} \in\left\{Y_{\alpha^{+}}, Y_{\kappa}\right\}$ and apply 7.3. 
Example 7.5. Let $\kappa$ be supercompact and let $\kappa \leq \lambda$ such that $2^{\lambda}=\lambda^{+}$.

(a) There are spaces $\left\{X_{\alpha}: \alpha<\lambda\right\}$ such that $X=\sum\left\{X_{\alpha}: \alpha<\lambda\right\}$ has a remote point $p \in v X$. We can have $\kappa_{p}=\kappa, \lambda_{p}=\lambda$ and $w_{p}$ any regular cardinal between $\kappa$ and $\lambda$.

(b) For each $\lambda^{\prime} \geq \lambda$, there are spaces $\left\{X_{\alpha}: \alpha<\lambda^{\prime}\right\}$ such that $X=$ $\sum\left\{X_{\alpha}: \alpha<\lambda^{\prime}\right\}$ has a remote point $p \in v X$ such that $\kappa_{p}=\kappa, \lambda_{p}=\left(\lambda^{\prime}\right)^{<\kappa}$ and $\kappa \leq w_{p} \leq \lambda$.

The example in (a) is obviously the same as that for 7.4. For (b) we do not assume that $2^{\lambda^{\prime}}=\left(\lambda^{\prime}\right)^{+}$hence we cannot use 7.3 directly. Let $\mathscr{U}$ be any $\kappa$-complete normal fine ultrafilter on $\left[\lambda^{\prime}\right]^{<\kappa}$. For each $a \in\left[\lambda^{\prime}\right]^{<\kappa}$, let $X_{a}$ be a space with a remote point $p_{a} \in v X_{a}$ (by part (a)). We let $p \in v X$ be the " $\mathscr{U}$-limit" of the collection $\left\{p_{a}: a \in\left[\lambda^{\prime}\right]^{<\kappa}\right\}$. That is, for $Z \in \mathscr{Z}(X), Z \in p$ iff $\left\{a \in\left[\lambda^{\prime}\right]^{<\kappa}: Z \cap X_{a} \in p_{a}\right\} \in \mathscr{U}$.

Remark 7.6. The result that $\kappa_{p} \leq w_{p}$ can be improved to $\left\{a \in[\lambda]^{<\kappa}:|a|<\right.$ wn $\left.\left(X_{a}\right)\right\} \in \mathscr{U}$ in 7.3 where each $X_{a}$ is pseudo- $\mu_{0}$-compact (this is needed by 7.5(b)). However I do not know if this suffices (i.e. replace $G\left(X_{a}\right)>|a|$ by $\operatorname{wn}\left(X_{a}\right)>|a|$ in 7.3).

\section{REFERENCES}

[B1] J. E. Baumgartner, Results and independence proofs in combinatorial set theory, Ph.D. thesis, Univ. of Calif., Berkeley, 1970.

[B2] __, Sacks forcing and the total failure of Martin's Axiom, preprint.

[B3] _ Iterated forcing, Surveys in Set Theory, edited by A. R. D. Mathias, Cambridge Univ. Press, 1983.

[BL] J. E. Baumgartner and R. Laver, Iterated perfect-set forcing, Ann. Math. Logic 17 (1979), 271-288.

[Be] M. G. Bell, Compact ccc non-separable spaces of small weight, Topology Proc. 5 (1980), 11-25.

[Br] $\mathrm{R}$. Blair, $A$ note on remote points, preprint.

[CS] S. B. Chae and J. H. Smith, Remote points and G-spaces, Topology Appl. 11 (1980), 243-246.

[ChKe] C. C. Chang and H. J. Keisler, Model theory, North-Holland, New York, 1977.

[vD1] E. K. van Douwen, Existence and applications of remote points, Bull. Amer. Math. Soc. 841 (1978), 161-163.

[vD2] _ Remote points, Dissertationes Math. 188 (1981).

[vD3] __, Covering and separation properties of box products, Surveys in General Topology, edited by G. M. Reed, Academic Press, 1980, pp. 55-130.

[vDvM] E. K. van Douwen and J. van Mill, Spaces without remote points, Pacific J. Math. 105 (1983), 69-75.

[D1] A. Dow, Weak P-points in compact ccc F-spaces, Trans. Amer. Math. Soc. 269 (1982), 557565.

[D2] __, Some separable spaces and remote points, Canad. J. Math. 34 (1982), 1378-1389.

[D3] _ _ Products without remote points, Topology Appl. 15 (1983), 239-246.

[D4] _ Remote points in spaces with $\pi$-weight $\omega_{1}$, Fund. Math. 124 (1984), 197-205.

[D5] _ _, Some linked subsets of posets, Israel J. Math. 59 (1987), 353-376. 
[DP] A. Dow and T. J. Peters, Game strategies yield remote points, Topology Appl. 27 (1987), 245-256.

[DvM] A. Dow and J. van Mill, On nowhere dense ccc P-sets, Proc. Amer. Math. Soc. 80 (1980), 697-700.

[DTW] A. Dow, F. D. Tall and W. A. R. Weiss, New proofs of the normal Moore space conjecture, preprint.

[F1] W. G. Fleissner, The normal Moore space conjecture and large cardinals, Handbook of SetTheoretic Topology, edited by K. Kunen and J. E. Vaughan, North-Holland, Amsterdam, 1984, pp. 733-760.

[F2] __ Homomorphism axioms and lynxes, Axiomatic Set Theory, Edited by J. E. Baumgartner, D. A. Martin and S. Shelah, Contemp. Math., vol. 31, Amer. Math. Soc., Providence, R.I., 1984, pp. 79-97.

[FG] N. J. Fine and L. Gillman, Remote points in $\beta R$, Proc. Amer. Math. Soc. 13 (1962), 29-36.

[G1] C. L. Gates, A study of remote points of metric spaces, Ph.D. thesis, Univ. of Kansas, 1973.

[G2] __ Some structural properties of the set of remote points of a metric space, Canad. J. Math. 32 (1980), 195-209.

[G3] __ A characterization of co-absoluteness for a class of metric spaces, Proc. Amer. Math. Soc. 80 (1980), 499-504.

[GJ] L. Gillman and M. Jerison, Rings of continuous functions, Springer-Verlag, New York, 1976.

[KaMa] A. Kanamori and M. Magidor, The evolution of large cardinals in set theory, Lecture Notes in Math., vol. 699, 1978, pp. 99-275.

[K1] K. Kunen, Set theory, North-Holland, Amsterdam, 1980.

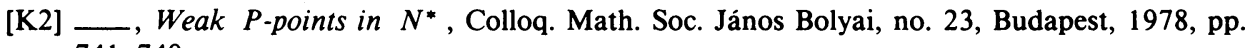
741-749.

[KvMM] K. Kunen, J. van Mill and C. F. Mills, On nowhere dense closed P-sets, Proc. Amer. Math. Soc. 78 (1980), 119-123.

[vM1] J. van Mill, Weak P-points in compact F-spaces, Topology Proc. 4 (1979), 609-628.

[vM2] __, Weak P-points in Cech-Stone compactifications, Trans. Amer. Math. Soc. 273 (1982), 657-678.

[P] T. J. Peters, Remote points, products and G-spaces, Ph.D. thesis, Wesleyan Univ., 1982.

[PoW] J. Porter and R. G. Woods, Nowhere dense subsets of metric spaces with applications to Stone-Cech compactifications, Canad. J. Math. 24 (1972), 622-630.

[R] W. Rudin, Homogeneity problems in the theory of Cech compactifications, Duke J. Math. 29 (1956), 409-419.

[Ru] M. E. Rudin, Lectures on set-theoretic topology, CBMS Regional Conf. Ser. in Math., no. 23, Amer. Math. Soc., Providence, R.I., 1975.

[S] S. Shelah, Proper forcing, Lecture Notes in Math., vol. 940, Springer-Verlag, 1982.

[T] F. D. Tall, Normality versus collectionwise normality, Handbook of Set-Theoretic Topology, edited by K. Kunen and J. E. Vaughan, North-Holland, Amsterdam, 1984.

[Te] T. Terada, On remote points in $v X-X$, Proc. Amer. Math. Soc. 77 (1979), 264-266.

[VWa] J. Vermeer and E. Wattel, Remote points, far points and homogeneity of $X^{*}$, Topology Structure II, Math. Centre Tracts, 116, Math. Centrum, Amsterdam, 1979, pp. 285-290.

[Wi] S. Williams, Box products, Handbook of Set-Theoretic Topology, edited by K. Kunen and J. E. Vaughan, North-Holland, Amsterdam, 1984.

[W1] R. G. Woods, Some $\aleph_{0}$-bounded subsets of Stone-Čech compactifications, Israel J. Math. 9 (1971), 250-256.

[W2] __, A Boolean algebra of regular closed subsets of $\beta X-X$, Trans. Amer. Math. Soc. 154 (1971), 23-36. 
[W3] _ Homeomorphic sets of remote points, Canad. J. Math. 23 (1971), 495-502.

[W4] _ Co-absolutes of remainders of Stone-Cech compactifications, Pacific J. Math. 37 (1971), 545-560.

[W5] _ _ A survey of absolutes of topological spaces, Topology Structure II, Math. Centre Tracts, 116, Math. Centrum, Amsterdam, 1979, pp. 323-362.

Department of Mathematics, University of Toronto, Toronto, Ontario M5S 1A1, CanAda

Current address: Department of Mathematics, York University, North York, Ontario M3J 1P3, Canada 